\title{
Herramientas de Screening del Desarrollo Psicomotor en Latinoamérica
}

\author{
AGUSTINA VERICAT ${ }^{1}$, ALICIA B. ORDEN $^{1,2}$ \\ 1. Instituto de Desarrollo e Investigaciones Pediátricas "Prof. Dr. Fernando E. Viteri" (IDIP-MS/CIC, PBA) \\ Hospital de Niños Sor M. Ludovica. La Plata, Buenos Aires, Argentina. \\ 2. Consejo Nacional de Investigaciones Científicas y Técnicas (CONICET).
}

\begin{abstract}
Psychomotor Development Screening Tools in Latin America

Primary Care physicians play an important role in detecting psychomotor development problems. There are a number of useful tools available for this purpose. This paper provides a review of the literature of some methods utilized in Latin America, with special emphasis on screening tools. A follow up protocol for patients with developmental delay is suggested in order to simplify the diagnostic process. Finally, a few hurdles in implementation of developmental control are pointed out.
\end{abstract}

(Key words: Psychomotor development, screening tools, primary care, Latin America).

Rev Chil Pediatr 2010; 81 (5): 391-401

\section{RESUMEN}

El médico que trabaja en atención primaria cumple un papel fundamental en la detección de los problemas del desarrollo psicomotor. Actualmente se dispone de una gran cantidad de herramientas útiles para la pesquisa de este tipo de trastornos. En este artículo se presenta una revisión actualizada de algunos de los métodos de evaluación del desarrollo psicomotor más frecuentemente utilizados en América Latina, con especial énfasis en las técnicas de screening. También se presenta un esquema de seguimiento de los pacientes con retraso psicomotor a fin de simplificar el proceso diagnóstico y, por último se plantean algunas dificultades para implementar la vigilancia del desarrollo.

(Palabras clave: Desarrollo psicomotor, tamizaje, atención primaria, Latinoamérica).

Rev Chil Pediatr 2010; 81 (5): 391-401

Trabajo recibido el 13 de marzo de 2010, devuelto para corregir el 28 de marzo de 2010, segunda versión el 10 de mayo de 2010, tercera versión el 16 de julio de 2010, aceptado para publicación el 05 de agosto de 2010.

Correspondencia a:

Dra. Agustina Vericat

E-mail: avericat77@yahoo.com.ar 


\section{Introducción}

El desarrollo psicomotor es la progresiva adquisición de habilidades funcionales del niño a medida que éste crece. Es un proceso gradual, en el cual es posible identificar etapas o estadios de creciente nivel de complejidad. Está determinado por aspectos biológicos, la interacción social y las experiencias propias del aprendizaje.

La Academia Americana de Pediatría define como problemas del desarrollo a todos aquellos cuadros crónicos y de inicio precoz que tienen en común la dificultad en la adquisición de habilidades motoras, de lenguaje, sociales o cognitivas que provocan un impacto significativo en el progreso del desarrollo de un niño ${ }^{1}$. Se presentan cuando existe fracaso en el logro de las conductas esperadas para una determinada edad ${ }^{2}$. Si bien algunos problemas del desarrollo pueden ser transitorios, los retrasos en la edad temprana pueden estar asociados a posteriores discapacidades, tales como retardo mental, parálisis cerebral, autismo, alteraciones del lenguaje y problemas del aprendizaje.

Se ha estudiado ampliamente la relación entre las influencias biológicas y medioambientales sobre el desarrollo madurativo. Entre los factores biológicos de riesgo pueden citarse prematurez, síndromes genéticos, asfixia perinatal, hiperbilirrubinemia neonatal e infecciones prenatales; mientras que desnutrición, analfabetismo materno, desocupación paterna, e inaccesibilidad al sistema de salud son considerados como factores de riesgo socioambiental ${ }^{2}$.

Se conocen "períodos críticos" durante los cuales hay una mayor susceptibilidad y vulnerabilidad a los diversos factores de riesgo. Existe consenso respecto a la ubicación de estos períodos críticos durante la etapa intrauterina y los dos o tres primeros años de vida, donde el organismo en general y particularmente el sistema nervioso central, presentan un alto ritmo de crecimiento y diferenciación $^{3-6}$. La mayor plasticidad cerebral ocurre durante la niñez temprana, de modo que en este período pueden realizarse intervenciones con resultados positivos. Si bien los pacientes con retrasos mentales severos tienen respues- tas más moderadas, se ha informado que la estimulación temprana tiene efectos positivos en los niños con retrasos leves o sutiles ${ }^{7}$.

Ante la necesidad de encontrar una metodología diagnóstica para este tipo de problemas, surgen como respuesta diferentes acciones que pueden llevarse a cabo en el consultorio. Por una parte, a través de la vigilancia del desarro1lo (development surveillance), que Dwornik ${ }^{8}$ definió como un proceso flexible y continuo en donde los profesionales capacitados llevan a cabo observaciones sistemáticas de los niños, durante el control de salud y engloba todas las actividades relacionadas con la promoción del desarrollo normal y la detección de problemas del desarrollo en la atención primaria de salud (APS). Por otra parte, el screening o pesquisa es una herramienta que se utiliza en el seguimiento del desarrollo de niños aparentemente normales con el objetivo de identificar a aquellos con alto riesgo de sufrir alteraciones de desarrollo9. Finalmente, la evaluación del desarrollo es una investigación más detallada de los niños en los que se sospechan problemas del desarrollo.

En Latinoamérica, la evaluación del desarrollo suele desarrollarse en forma asistemática. Esto se atribuye en general, a recursos escasos, tanto materiales como al tiempo disponible para realizar dichas pruebas en el nivel primario de atención ${ }^{10}$. En 1988, un estudio realizado por Lira y Gálvez en Chile, informaba que sólo el $50 \%$ de los médicos de atención primaria utilizaban la escala chilena EEDP y lo hacía sólo una vez en cada paciente ${ }^{11}$. Ésto se ha ido modificando con el tiempo ${ }^{12}$. La creciente sobrevida de niños prematuros, de bajo peso al nacer y de pacientes con ciertas enfermedades graves, genera nuevas formas de morbilidad $^{13}$, donde la evaluación de los problemas del desarrollo demanda una inclusión en la práctica clínica cotidiana.

El presente artículo realiza una revisión bibliográfica general de algunos de los métodos de evaluación de los problemas del desarrollo psicomotor más frecuentemente utilizados en América Latina, con especial énfasis en las técnicas de screening. Esta revisión intenta además desarrollar un esquema de seguimiento de los pacientes con retraso psicomotriz a 
fin de simplificar el proceso diagnóstico. Con este propósito se consultaron libros de texto específicos y las bases SciELO, LILACS y Medline.

\section{Papel del pediatra en la detección de problemas del desarrollo}

El pediatra es quien habitualmente sigue el crecimiento del niño durante sus primeros años de vida, quien interactúa con su familia y conoce su medioambiente. Dado que este seguimiento puede prolongarse hasta la adolescencia, se transforma en alguien idóneo para observar el desarrollo. Desempeña una función preventiva en este campo, aunque su papel no sólo se limita a la realización de un diagnóstico precoz, sino que también se extiende a la asistencia y contención del niño y su familia ${ }^{14}$. De él se espera que tenga un conocimiento vasto en el campo del desarrollo infantil, cuente con el tiempo y las herramientas necesarias, conozca los recursos disponibles en su comunidad para tratar esos problemas y sepa coordinar con especialistas el tratamiento a seguir? ${ }^{7}$.

El Comité de Discapacidad de la $\mathrm{AAP}^{15}$ recomendó en un informe que la vigilancia del desarrollo debe ser incorporada a la consulta de niño sano. Cualquier duda que surja durante la evaluación de los niños deberá ser abordada con prontitud. Además de esta pesquisa informal, se recomienda aplicar tests estandarizados a todos los niños de 9,18 y 30 meses de edad (esta última puede realizarse a los 24 meses junto al control de salud de dicha edad).

Ante la detección de un retraso madurativo, comienza un proceso de búsqueda de resolución del problema que podría denominarse recorrido terapéutico. Éste contempla diferentes niveles de atención que no corresponden exactamente con las clasificaciones basadas en la complejidad de la atención médica (tabla 1):

Tabla 1. Recorrido terapéutico del paciente con trastornos del desarrollo

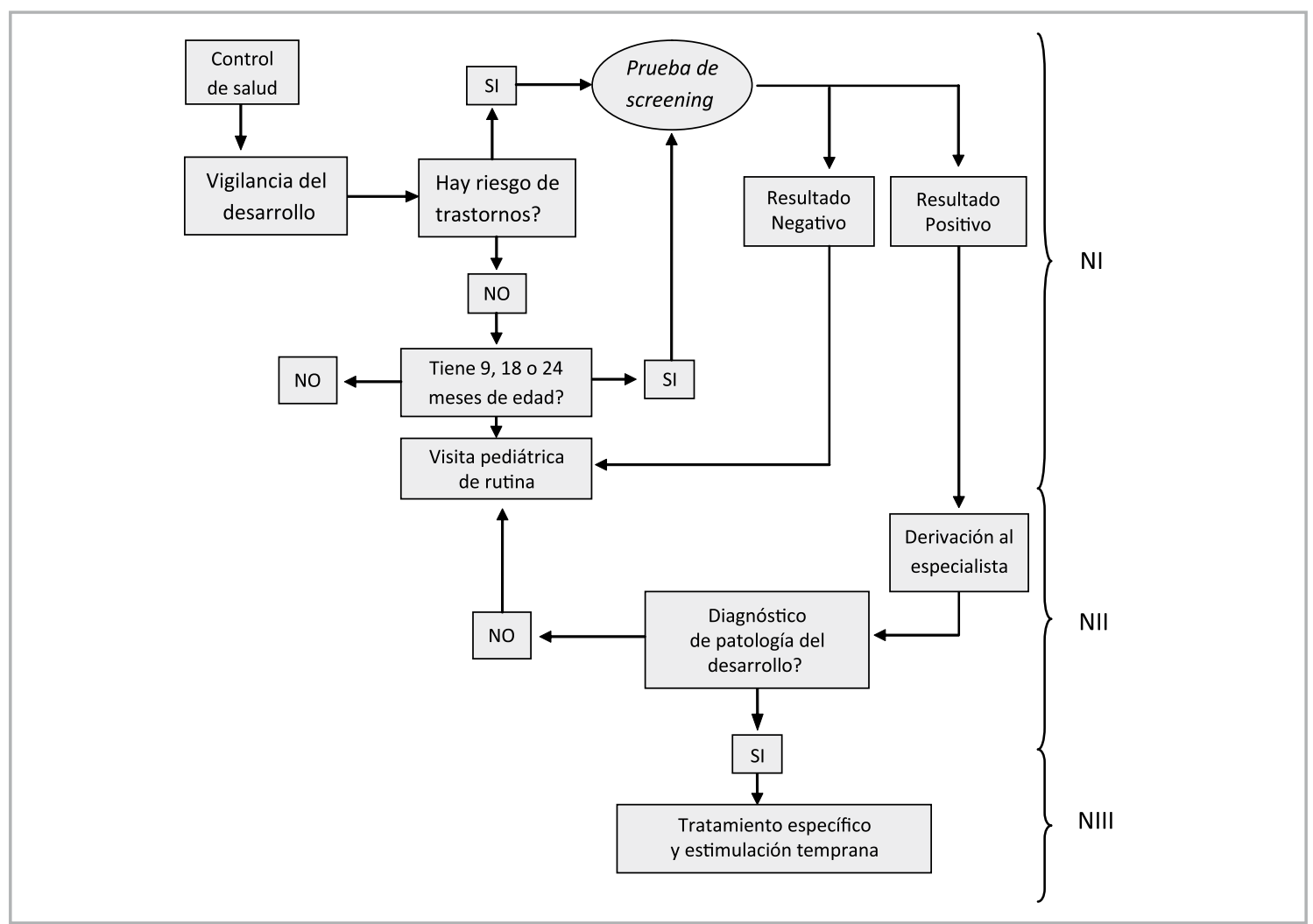

Fuente: adaptado de AAP, 2006 (13) 
- Nivel I: engloba las llamadas acciones de atención primaria de la salud llevadas a cabo por el pediatra o médico en el consultorio y por docentes con cierto entrenamiento en el ámbito escolar.

- Nivel II: incluye acciones desarrolladas por especialistas en desarrollo neurológico infantil, entrenados en técnicas psicométricas más complejas.

- Nivel III: abarca el tratamiento específico de la patología (estimulación temprana, rehabilitación, terapia cognitivo-conductual, etc).

Tabla 2. Principales técnicas de screening para detectar problemas del desarrollo psicomotor utilizadas en Latinoamérica

\begin{tabular}{|c|c|c|c|c|c|c|}
\hline $\begin{array}{l}\text { Tipo de } \\
\text { prueba }\end{array}$ & Denominación & Autor / año / país & Validez & $\begin{array}{l}\text { Rango } \\
\text { de edad }\end{array}$ & Aspectos que evalúa & $\begin{array}{l}\text { Refe- } \\
\text { rencia }\end{array}$ \\
\hline $\begin{array}{l}\text { Encuestas para } \\
\text { Padres }\end{array}$ & $\begin{array}{l}\text { ASQ (Age and Stages } \\
\text { Questionnaire) }\end{array}$ & $\begin{array}{l}\text { Bricker et al, 1995; } \\
\text { Squires et al, } 2009 . \\
\text { EE.UU. }\end{array}$ & $\begin{array}{l}\text { S: } 0,86 \\
E: 0,85\end{array}$ & 1 a 5 años & $\begin{array}{l}\text { Comunicación, motrici- } \\
\text { dad gruesa, motricidad } \\
\text { fina, resolución de } \\
\text { problemas, lenguaje, } \\
\text { personal social }\end{array}$ & 20 \\
\hline \multirow[t]{10}{*}{$\begin{array}{l}\text { Pruebas de } \\
\text { screening }\end{array}$} & Test de Denver II & $\begin{array}{l}\text { Frankenburg y } \\
\text { Dodds, 1967; } 1992 . \\
\text { EE.UU. }\end{array}$ & $\begin{array}{l}\text { S: } 0,56-0,83 \\
E: 0,43-0,80\end{array}$ & 0 a 6 años & $\begin{array}{l}\text { Motricidad gruesa, } \\
\text { lenguaje, motricidad } \\
\text { fina-adaptativa, personal } \\
\text { social }\end{array}$ & 23 \\
\hline & $\begin{array}{l}\text { CAT/CLAMS (Cogni- } \\
\text { tive Adaptative Test/ } \\
\text { Clinical Linguistic and } \\
\text { Auditory Milestone } \\
\text { Scale) }\end{array}$ & $\begin{array}{l}\text { Accardo y Capute, } \\
\text { 2005. EE.UU }\end{array}$ & $\begin{array}{l}\text { Bajo Riesgo } \\
\text { S: 0,21-0,67 } \\
\text { E: 0,95-1,00 } \\
\text { Alto Riesgo } \\
\text { S: 0,05-0,88 } \\
\text { E: 0,82-0,98 }\end{array}$ & $\begin{array}{l}1 \text { mes a } \\
3 \text { años }\end{array}$ & $\begin{array}{l}\text { Lenguaje, motricidad } \\
\text { fina y visual }\end{array}$ & 26 \\
\hline & $\begin{array}{l}\text { BINS (Bayley Infant } \\
\text { Neurodevelopmental } \\
\text { Screener) }\end{array}$ & $\begin{array}{l}\text { Bayley, 1969; } 1993 . \\
\text { EE.UU. }\end{array}$ & $\begin{array}{l}\text { S: } 0,75-0,86 \\
E: 0,75-0,86\end{array}$ & $\begin{array}{l}3 \text { meses a } \\
2 \text { años }\end{array}$ & $\begin{array}{l}\text { Funciones neurológicas } \\
\text { básicas, receptivas, } \\
\text { expresivas y procesos } \\
\text { cognitivos }\end{array}$ & 27 \\
\hline & $\begin{array}{l}\text { EEDP (Escala de Eva- } \\
\text { luación del Desarrollo } \\
\text { Psicomotor) }\end{array}$ & $\begin{array}{l}\text { Rodríguez et al, } \\
\text { 1978. Chile }\end{array}$ & $N D^{*}$ & 0 a 2 años & $\begin{array}{l}\text { Social, lenguaje, coordi- } \\
\text { nación y motricidad }\end{array}$ & 29 \\
\hline & $\begin{array}{l}\text { TEPSI (Test de Desa- } \\
\text { rrollo Psicomotor) }\end{array}$ & $\begin{array}{l}\text { Haeussler y } \\
\text { Marchant, } 1980 . \\
\text { Chile }\end{array}$ & $N D^{*}$ & 2 a 5 años & $\begin{array}{l}\text { Coordinación, motrici- } \\
\text { dad y lenguaje }\end{array}$ & 30 \\
\hline & $\begin{array}{l}\text { PRUNAPE (Prueba } \\
\text { Nacional de Pesquisa) }\end{array}$ & $\begin{array}{l}\text { Lejarraga et al, } \\
\text { 2005. Argentina }\end{array}$ & $\begin{array}{l}\text { S: } 0,80 \\
E: 0,93\end{array}$ & 0 a 6 años & $\begin{array}{l}\text { Motricidad fina, motri- } \\
\text { cidad gruesa, peronal } \\
\text { social y lenguaje }\end{array}$ & 31 \\
\hline & $\begin{array}{l}\text { EAIS (Escala Argen- } \\
\text { tina de Inteligencia } \\
\text { Sensorio Motriz) }\end{array}$ & $\begin{array}{l}\text { Oiberman et al, } \\
\text { 2002. Argentina }\end{array}$ & $N D^{*}$ & $\begin{array}{l}6 \text { meses a } \\
2 \text { años }\end{array}$ & Procesos cognoscitivos & 33 \\
\hline & $\begin{array}{l}\text { EDIN (Escala del } \\
\text { Desarrollo Integral } \\
\text { del Niño) }\end{array}$ & $\begin{array}{l}\text { Atkin et al, } 1987 . \\
\text { México }\end{array}$ & $N D^{*}$ & 0 a 6 años & $\begin{array}{l}\text { Motricidad fina, motri- } \\
\text { cidad gruesa, reflejos, } \\
\text { lenguaje, socio-emocio- } \\
\text { nal y cognitiva }\end{array}$ & 34 \\
\hline & $\begin{array}{l}\text { Escala Abreviada del } \\
\text { Desarrollo }\end{array}$ & $\begin{array}{l}\text { Ortíz Pinilla, } 1991 . \\
\text { Colombia }\end{array}$ & $N D^{*}$ & $\begin{array}{l}3 \text { meses a } \\
5 \text { años }\end{array}$ & $\begin{array}{l}\text { Motricidad fina-adapta- } \\
\text { tiva, motricidad gruesa, } \\
\text { reflejos, audición-len- } \\
\text { guaje y personal social }\end{array}$ & 6 \\
\hline & $\begin{array}{l}\text { Escala de Desenvol- } \\
\text { vimento de Heloise } \\
\text { Marihno (EDHM) }\end{array}$ & $\begin{array}{l}\text { Marihno, } 1977 . \\
\text { Brasil }\end{array}$ & $N D^{*}$ & 0 a 9 años & $\begin{array}{l}\text { Desarrollo físico, mental } \\
\text { y social }\end{array}$ & 35 \\
\hline
\end{tabular}

S: sensibilidad, E: especificidad, *No determinado. Fuente: elaboración propia. 


\section{Técnicas de evaluación del desarrollo psicomotor}

Los métodos para evaluación del desarrollo son numerosos y variados, incluyendo encuestas a los padres, observación directa en el consultorio del pediatra, pruebas de screening y escalas del desarrollo (tabla 2). Actualmente existen numerosas pruebas que pueden ser utilizadas en APS, en particular en el consultorio pediátrico. Una vez que sospecha un problema, el médico debe insistir en llegar a un diagnóstico. Es aquí donde, dentro de un abordaje mucho más amplio, intervienen el psicólogo, psiquiatra infantil, neurólogo y otros especialistas.

La evaluación del desarrollo engloba fundamentalmente tres tipos de acciones: vigilancia, pruebas de screening y evaluación del desarrollo. La vigilancia del desarrollo es un método sencillo e informal, con buena respuesta, si quien la realiza posee los conocimientos suficientes sobre desarrollo infantil. Su punto débil radica en que pone énfasis en el juicio clínico del pediatra, en su experiencia y en su visión subjetiva ${ }^{16}$. En general este método detecta los problemas severos, pero en menor medida los retrasos sutiles del desarrollo.

En el otro extremo, la evaluación del desarrollo propiamente dicha ${ }^{9}$, es llevada a cabo por diferentes especialistas, como neurólogos, psicopedagogos, psiquiatras, etc, aplicando técnicas diagnósticas más complejas como son los tests de inteligencia y métodos complementarios de estudio, implicando el Nivel III de atención (tabla 1). Mediando entre ambos tipos de acciones se encuentran las pruebas de pesquisa o screening del desarrollo.

\section{Pruebas de screening del desarrollo}

Detectan en forma rápida y sencilla posibles anomalías del desarrollo en niños pequeños. Este tipo de pruebas deben poseer consistencia (estabilidad prueba-reprueba), validez, alta sensibilidad y especificidad, ser socialmente aceptables, sencillas, rápidas y de bajo costo. Incluyen encuestas para padres y pruebas aplicadas a los niños o de screening propiamente dichas.

\section{a. Encuestas para padres}

Aunque se encuentran traducidas al español, son usadas más frecuentemente en países del hemisferio norte, en especial cuando los padres presentan inquietudes respecto a la maduración de sus hijos. Muchos datos son sumamente útiles (por ej. si el niño se para solo, si controla esfínteres, qué palabras dice, etc). Pertenecen a este tipo de entrevistas el ASQ (Age and Stages Questionnaire) ${ }^{17}$, el CDI (Child Development Inventory) ${ }^{18}$ y el PEDS (Parent's Evaluation of Developmental Status) ${ }^{19}$. El CDI evalúa niños de 1,5 a 6 años de edad a través de las áreas motricidad gruesa, motricidad fina, lenguaje expresivo y comprensión del lenguaje, así como el desarrollo social, la autonomía y el desarrollo en general. Se correlaciona significativamente con pruebas muy utilizadas como CAT/CLAMS y test de Bayley II) ${ }^{18}$. El ASQ evalúa niños de 1 a 5,5 años en las áreas de comunicación, motricidad gruesa y fina, resolución de problemas, lenguaje y personal social, entre otras ${ }^{17,20}$. El PEDS evalúa problemas del desarrollo y comportamiento de niños de 0 a 8 años ${ }^{19}$. Las experiencias más conocidas en Latinoamérica han sido realizadas en Chile utilizando el $\mathrm{ASQ}^{21}$.

\section{b. Pruebas de screening}

Son fáciles de administrar y requieren relativamente poco entrenamiento por lo que son ideales para ser usadas por el pediatra en su práctica diaria. Las más conocidas son las de Denver, CAT/CLAMS y BINS que fueron realizadas en EE.UU y son de uso frecuente en Latinoamérica. Sin embargo, muchos países de la región disponen de pruebas propias, tales como EEDP, TEPSI y PRUNAPE, entre las más conocidas ${ }^{22}$.

b.1. Test de Denver II. Fue ideado para proporcionar un método breve de detección de problemas del desarrollo, estandarizado en 1036 lactantes y preescolares ${ }^{23,24}$. Analiza cuatro áreas: motricidad gruesa, lenguaje, motricidad fina-adaptativa y personal-social. La evaluación del desarrollo se basa en el desempeño del niño durante la entrevista y por datos referidos por los padres. El resultado mide si el niño cumple o no los ítems esperados para su 
edad. En base a esto, se lo clasifica como normal, sospechoso o con retraso del desarrollo y en este último caso debe ser derivado a un especialista (neurólogo infantil, psicopedagogo, etc) para su seguimiento. Su sensibilidad y especificidad son moderadas a bajas ${ }^{15}$.

b.2. CAT/CLAMS: (Cognitive Adaptative Test/ Clinical Linguistic and Auditory Milestone Scale). Es un método basado en el test de Bayley, desarrollado para ser utilizado en el consultorio del pediatra. Evalúa el desarrollo de niños entre 1 y 36 meses en las áreas lenguaje, motricidad fina y visual. Permite comparar las habilidades lingüísticas y no lingüísticas para la resolución de problemas y por tanto, ayuda en el diagnóstico y la derivación adecuada $^{25}$. Consta de una batería de lenguaje: CLAMS (Clinical Linguistic \& Auditory Milestone Scale) que mide tanto los hitos de lenguaje receptivo como expresivo y de una batería de resolución de problemas visomotores: CAT (Cognitive Adaptive Test) que evalúa los hitos visomotores. El test tiene un tiempo de aplicación de 10 a 15 minutos y otorga un cociente de desarrollo ${ }^{26}$.

b.3. BINS (Bayley Infant Neurodevelopmental Screener). Es una prueba basada en las escalas Bayley de desarrollo infantil BSID-II, que evalúa niños de 3 a 24 meses de edad $^{27}$. Valora las funciones neurológicas básicas, las receptivas, las expresivas y los procesos cognitivos. Se utiliza para clasificar a los niños como de riesgo alto, moderado o bajo de presentar retrasos de desarrollo. Mientras que el test de Bayley requiere un examinador altamente entrenado e insume unos 30 a 60 minutos el BINS puede ser administrado por un profesional capacitado en un lapso corto de tiempo, por lo que es costo-efectiva para el screening de rutina ${ }^{28}$. Los valores de sensibilidad y especificidad son moderados a altos ${ }^{15}$.

b.4. EEDP (Escala de Evaluación del Desarrollo Psicomotor). Es una herramienta de origen chileno para ser empleada en niños desde el nacimiento hasta los 24 meses. Constituye el primer instrumento de este tipo estandarizado de ese país y cuenta con diferentes baremos según el nivel socioeconómico. Es de fácil aplicación y puede ser administrado tanto por profesionales especializados como no especializados, pudiendo utilizarse como parte de un programa de estimulación psicosocial junto al control de los niños sanos. Las áreas evaluadas en esta prueba son: social, lenguaje, coordinación y motricidad, obteniéndose un coeficiente de desarrollo. Se estudió la confiabilidad del instrumento (test-retest), obteniéndose alta consistencia en sus ítems ${ }^{29}$.

b.5. TEPSI (TEST DE DESARROLLO PSICOMOTOR). Fue confeccionado en 1980 por Haeussler y Marchant en Chile, como instrumento para los profesionales de educación preescolar y ha sido empleado en varios países de Latinoamérica. Es un test que evalúa niños de 2 a 5 años en las áreas de coordinación, motricidad y lenguaje. En 1993, se realizó una versión para ciegos ${ }^{30}$.

b.6. PRUNAPE (Prueba Nacional de Pesquisa). Es una prueba argentina aplicable en niños de 0 a 6 años de edad. La prueba consta de setenta y nueve pautas madurativas pertenecientes a las áreas motriz fina y gruesa, lenguaje y personal-social. Tiene una duración de aproximadamente 15 minutos y consiste en solicitar al niño que realice una serie de ejercicios con papel y lápiz, cubos, tarjetas dibujadas, que varían según la edad. En los niños menores de dos años que nacieron antes de las 37 semanas de edad gestacional se debe realizar un ajuste de la edad por la prematurez. La PRUNAPE fue sometida a un proceso de validación, estimándose la sensibilidad y la especificidad. Se trata de un test compatible con las costumbres culturales locales ${ }^{31}$.

b.7. EAIS (Escala de Inteligencia Sensorio Motriz). Es una escala desarrollada en Argentina, que evalúa los procesos cognoscitivos en niños de 6 meses a 2 años. A diferencia de otras escalas, incorpora aspectos cualitativos, al considerar tanto el logro final alcanzado como el modo de resolución de la situación propuesta. Es una adaptación de la Escala de Casati Lézine, basada en la teoría de Piaget ${ }^{32}$ para quien la inteligencia es esencialmente operativa: su función es actuar sobre los materiales 
que ella recibe, movilizarlos y transformarlos. La EAIS fue realizada a partir de una muestra poblacional de 323 bebés argentinos de diferentes estratos sociales de la ciudad de Buenos Aires y del Gran Buenos Aires. Actualmente se está intentando realizar la estandarización a nivel nacional ${ }^{33}$.

b.8. EDIN (Escala del Desarrollo Integral del Niño). Es una prueba realizada en Costa Rica, que evalúa la motricidad fina y gruesa, los reflejos, el lenguaje y las áreas socioemocional y cognitiva en niños de 0 a 6 años además obtener información adicional sobre hábitos del niño. Contiene propiedades psicométricas adecuadas, además de realizar una comparación entre diversos niveles socioeconómicos. Fue diseñada para proporcionar mejores descripciones del desarrollo del niño que las que ofrecen otras escalas estandarizadas y elaboradas en otros países. Sin embargo, no hay datos disponibles en cuanto a la validez concurrente y predictiva ${ }^{34}$.

b.9. Escala Abreviada de Desarrollo. Es una escala colombiana que evalúa niños de 3 meses a 5 años de edad, a partir de cuatro áreas: motricidad fina-adaptativa, motricidad gruesa, audición y lenguaje y área personal social. De su aplicación se obtienen cuatro posibles resultados que van desde el nivel de alerta hasta el nivel alto de sospecha de problemas del desarrollo ${ }^{6}$.

b.10. Escala de Desenvolvimento de Heloise Marihno (EDHM). Esta escala de origen brasilero puede ser utilizada en niños de 0 a 9 años. Evalúa las áreas de desarrollo físico, mental y social, otorgando un coeficiente de desarrollo ${ }^{35}$.

\section{Discusión}

Dependiendo de su finalidad, existen variados procedimientos que cumplen diferentes funciones en el estudio del desarrollo infantil. Para el seguimiento periódico e individual del niño, se suele utilizar la vigilancia del desarro1lo, que se realiza en forma rutinaria en el control de salud. Cuando el objetivo es identificar a niños en situación de riesgo de presentar un problema del desarrollo pueden aplicarse las pruebas de screening, que realizan un primer tamizaje. En los casos en que hay una necesidad diagnóstica, se hace indispensable la evaluación por parte de especialistas que trabajen en forma multidisciplinaria. Aunque estos procedimientos puedan explicarse en forma individual, en la práctica se hallan comúnmente interconectados, lo que permite obtener mejores resultados 9 .

Numerosas sociedades científicas recomiendan la vigilancia y el screening del desarrollo en la consulta pediátrica. Si bien se reconoce la importancia que tiene el diagnóstico precoz, son pocos los médicos que logran llevar a cabo tales acciones. ¿Por qué ocurre esto? Por una parte, muchos pediatras y médicos de atención primaria tienen conocimientos insuficientes y/o creencias equivocadas sobre desarrollo madurativo normal ${ }^{36,37}$. En general, los médicos suelen derivar a aquellos niños con retrasos más severos y a los de mayor edad, a pesar de que existe clara evidencia de los beneficios de la intervención precoz de los niños con signos "blandos" o sutiles ${ }^{15}$. También suele ocurrir que se otorga mayor importancia al cumplimiento de hitos de motricidad gruesa, como caminar y sentarse, pero no tanto a hitos del lenguaje o del área personal social. Se sabe que los niños con retraso motor aislado son derivados a especialistas para diagnóstico a edades más tempranas que los niños con retraso generalizado o aquellos con retraso del lenguaje $^{36}$. Según Álvarez Gómez et al ${ }^{7}$, la misma terminología "retraso psicomotor" indica hasta qué punto se acentúa lo "motor" siendo este aspecto peor predictor del desarrollo que aquellas otras capacidades que están cargadas con más intencionalidad y con más significación cognitiva o relacional que el tono o los hitos puramente motrices y que los pediatras están menos acostumbrados a valorar.

Finalmente, otro factor que obstaculiza la implementación de la vigilancia sistemática del desarrollo es el tiempo como recurso escaso en el nivel primario de atención, teniendo en cuenta que en un control de salud de alrededor de media hora se debe interrogar a los padres, darles pautas de alarma, educarlos sobre 
medidas higiénico dietéticas, pesar y medir al niño y realizar otros actos médicos ${ }^{10}$. Así, en un estudio realizado en Brasil, los médicos de atención primaria consideraban que las escalas del desarrollo eran muy complejas y demandaban mucho tiempo para ser tomadas ${ }^{37}$.

En Latinoamérica, se cuenta con varias pruebas de pesquisa que pueden utilizarse en APS (tabla 2). En el momento de tener que elegir una, el médico se encuentra con la incertidumbre de cuál es la más adecuada. Una consideración básica concierne al rango de edad de aplicación de una prueba. Las pruebas EDIN, PRUNAPE y Denver son aplicables desde el nacimiento hasta los seis años de edad. Otras en cambio, cubren rangos menores. Por ejemplo, la EEDP evalúa niños hasta los dos años y el TEPSI niños de dos a cinco años y la diferencia en su formato hace dificultoso coordinarlas como un solo instrumento ${ }^{38}$.

En general, todas evalúan cuatro áreas del desarrollo (personal social, motricidad fina y gruesa y lenguaje), pero algunas como CAT/ CLAMS no valoran motricidad gruesa, por lo cual deben complementarse con otra prueba. La EAIS, no mide las áreas por separado, sino que evalúa la esfera cognitiva en forma global. Con algunas excepciones como la prueba TEPSI, la mayoría no contempla déficits sensoriales, impidiendo la evaluación de niños con amaurosis o hipoacusia y tampoco posibilitan valorar a niños con minusvalías físicas como la amputación de miembros. Debe tenerse en cuenta que todas las pruebas parcializan la información. Como lo señala Ortiz Pinilla ${ }^{6}$ ninguna de ellas contempla el desarrollo infantil en forma exhaustiva, ya que todo instrumento presupone la evaluación de ciertas áreas en detrimento de otras.

La mayoría de los screening son de corta duración: la PRUNAPE insume 12 a $15 \mathrm{mi}-$ nutos, CAT/CLAMS 10 a 20 minutos, TEPSI 30 minutos y EEDP 7 a 15 minutos, entre algunos ejemplos ${ }^{39}$. Sin embargo, el tiempo de aplicación de cualquier prueba está asociado a varios factores, entre los que se incluyen el entrenamiento del evaluador, la edad del niño y su comportamiento durante la experiencia, la complejidad de la prueba y el contexto asistencial en el cual es desarrollada ${ }^{10,38}$.
En cuanto a los resultados, algunas pruebas dan un perfil de riesgo (PRUNAPE, DENVER etc), mientras otras otorgan un coeficiente de desarrollo, que es un valor numérico, como por ejemplo, la EEDP o CAT/CLAMS. Sin embargo, esta diferencia no es importante a fines prácticos, ya que el resultado de este tipo de evaluaciones es siempre una sospecha, no un diagnóstico.

En relación a la fiabilidad de los tests, muchos requieren de datos aportados por los progenitores, especialmente las encuestas para padres (ASQ, CDI o PEDS). Estas encuestas son una buena opción cuando el tiempo del profesional es muy limitado ${ }^{21}$. Sin embargo, debe tenerse en cuenta que a veces la información no es recordada en forma adecuada, pudiendo causar un sesgo de memoria. Las respuestas otorgadas por los padres están también condicionadas por sus conocimientos sobre el desarrollo infantil. En este sentido, algunos estudios han observado que un mayor conocimiento produce información más confiable y viceversa ${ }^{40}$. De esta forma, los padres pueden minimizar algunos hechos o malinterpretar ciertos signos, por ejemplo, referir que el niño dice "mamá" y "papá" porque dice " $m a$ ma ma... pa pa pa...", cuando en realidad emite sonidos no específicos. En algunos casos puede aparecer una negación parental no explícita en relación a algún problema percibido en el hijo, que puede "alterar" sus respuestas y -en otros casos- obviar algunos hechos que para el médico pueden ser valiosos. Esto puede darse dentro del proceso del duelo por la pérdida del niño sano, que a veces desencadena en la familia un peregrinaje profesional que les devuelva esa normalidad ${ }^{14}$. Esto implica tener en cuenta que la información de los padres, aunque valiosa, tiene siempre un grado variable de subjetividad.

Otro elemento fundamental a tener en cuenta en el momento de elección de una prueba es su validez. Para que una herramienta de screening sea válida en la detección de un problema específico debe cumplir con pautas mínimas de sensibilidad y especificidad así como de valores predictivos positivos y negativos ${ }^{16,40}$. Un problema común a algunas pruebas latinoamericanas es que sólo disponen de validez de 
constructo pero carecen de validez predictiva. En el caso de haber sido validadas es importante tener en cuenta que la sensibilidad y la especificidad no sean menores al $70 \%$. Una baja sensibilidad no detectará a muchos pacientes con problemas del desarrollo ${ }^{40}$ mientras que una alta sensibilidad puede sobrediagnosticar casos, sin embargo, el mayor riesgo sería no identificar a niños que presenten alguna dificultad ${ }^{16}$. Finalmente, si una prueba es poco específica identificará demasiados niños como sospechosos, provocando excesivas derivaciones a especialistas y sobrecargando al sistema de salud. En el caso de los cuestionarios para padres las opiniones son controvertidas. Algunos estudios han informado baja sensibilidad y especificidad para el ASQ y el $\mathrm{CDI}^{41}$ mientras otros hallaron valores moderados a altos para estos indicadores ${ }^{15}$.

Es importante elegir una prueba que sea coherente con las costumbres locales ${ }^{2}$. En Latinoamérica y en América del Sur en particular, se cuenta con este tipo de pruebas que fueron construidas con baremos locales. Idealmente, es bueno elegir un screening confeccionado con una población de características semejantes a la del niño que se quiere evaluar, ya que como lo afirman Tellería y Molina ${ }^{42}$, aunque el desarrollo sea un proceso universal, tiene rasgos propios de cada país o región, debido a la interacción de elementos de cada cultura en particular. Este es un problema común a la mayoría de las pruebas, dado que contienen elementos que no pueden considerarse transculturales ${ }^{12}$. Por ello, a pesar de que aparentemente han sido estandarizadas y son aplicables en diferentes contextos urbanos, no serían utilizables en poblaciones especiales (por ej. cuando se evalúa al niño mediante objetos que para él son desconocidos o tienen distinto significado en su cultura). En estos casos, si las pruebas no son utilizadas correctamente, más que medir el desarrollo sirven para predecir el éxito social ${ }^{6}$.

Los resultados de una prueba no deben emplearse para diagnosticar, por sí mismos, estados patológicos ${ }^{43}$. Deben interpretarse dentro de un marco conceptual, que tenga en cuenta al niño y su familia, al ambiente y la cultura, incluyendo al evaluador mismo. A pesar de la resistencia inicial del médico en incorporarlos al control de salud, los instrumentos de screening ahorran tiempo y recursos. Han venido a paliar en cierta forma la falta de organización y sistematización en el estudio del desarrollo psicomotor, siendo cada vez más adecuados y fáciles de utilizar ${ }^{8}$. Otorgan al profesional una herramienta fiable, de rápida aplicación que informa un resultado claro: pasa la prueba o no la pasa. En cierta forma, empujan al pediatra a derivar al niño si falla y comienzan a cambiar la idea tan arraigada de "esperar y ver qué pasa", acortando los tiempos de evaluación y sistematizando el proceso diagnóstico. Por otra parte, al ser instrumentos sencillos de aplicar requieren un corto tiempo de entrenamiento, pudiendo hacerse capacitaciones en jornadas, talleres y congresos.

Si una vez aplicadas la vigilancia del desarrollo o la prueba de screening dan como resultado que el niño tiene un posible problema del desarrollo, surge el cuestionamiento de cuáles son los pasos a seguir. Muchas veces esto es -en cierta forma- un trabajo artesanal, que varía según los recursos disponibles en el lugar de trabajo: mientras que algunos centros cuentan con profesionales médicos especializados en desarrollo, en otros ámbitos ese rol lo cumplen psicólogos, psicopedagogos o terapistas ocupacionales. Durante el proceso diagnóstico deberán realizarse estudios para descartar déficits sensoriales y fonológicos y buscar patologías asociadas a los problemas del desarrollo ${ }^{2}$. El diagnóstico de certeza a menudo exige conocimientos y experiencia que deben ser aportados por más de un profesional. En general, el pediatra es quien debe coordinar las intervenciones, acompañado por los algunos de los mencionados especialistas. Posteriormente, deben iniciarse acciones de intervención temprana $^{7,14}$. En algunas ocasiones no se llega a un diagnóstico de certeza hasta mucho tiempo después de iniciado el recorrido terapéutico, por lo que esperar esta instancia para realizar una intervención es desaprovechar tiempo muy valioso.

De esta manera se llega al final de un recorrido que muchas veces es largo y angustiante. Es responsabilidad del médico, independientemente del lugar que ocupe en el equipo de 
salud, que este recorrido sea lo más directo y sencillo posible, evitando que el niño y su familia circulen de consultorio en consultorio sin un orden establecido y posibilitando un diagnóstico precoz y un tratamiento oportuno.

\section{Conclusiones}

Los profesionales vinculados al seguimiento del desarrollo infantil, especialmente los pediatras, tienen un rol fundamental en la detección temprana de los problemas del desarrollo psicomotor, aplicando técnicas formales y sencillas de screening en su práctica diaria. En Latinoamérica se dispone de variados tests de pesquisa, lo que plantea la dificultad de cuál aplicar. No existe una prueba ideal, esto es, una que cumpla con todos los criterios de modo absoluto. Pero al menos dos criterios aparecen como elementales: uno se refiere a su validez y el otro al contexto de su aplicación. Esto implica la necesidad de sopesar sus ventajas y limitaciones, teniendo en cuenta los objetivos del estudio, el niño o grupo de población objeto del tamizaje y el contexto cultural donde ese instrumento es aplicado, entre los aspectos más importantes.

Utilizada en forma rutinaria, la pesquisa agiliza el proceso diagnóstico, motiva la charla con los padres, estimula al médico a formarse en el tema y, fundamentalmente, fortalece la relación médico-paciente, ya que es un espacio en el cual el profesional deja de actuar como tal para jugar con el niño y acercase a él. Pero esta tarea debe completarse con un trabajo multidisciplinario, a fin de derivar en forma temprana a esos niños para su diagnóstico de certeza y para la implementación de un tratamiento oportuno.

\section{Referencias}

1.- American Academy of Pediatrics Committee on Children with Disabilities: The pediatrician's role in development and implementation of an Individual Education Plan (IEP) and/or an Individual Family Service Plan (IFSP). Pediatrics 1999; 104: 124-7.

2.- Lejarraga $H$ : Enfoque pediátrico del desarrollo y sus problemas. En: Lejarraga H. ed. Desarrollo del Niño en
Contexto, Buenos Aires: Paidós. 2004; 341-78.

3.- Dobbing J: Vulnerable periods in developing brain. En: Applied Neurochemistry. Oxford: Blackwell 1968; 287316.

4.- Winick M, Fish I, Rosso P: Cellular recovery in rat tissues after a brief period of neonatal malnutrition. J Nutr 1968; 95: 623-6.

5.- Winick $M$, Rosso $P$ : Head circumference and cellular growth of the brain in normal and marasmatic children. J Pediatr 1969; 74: 774-8.

6.- Ortiz Pinilla N: Escala Abreviada de Desarrollo-EAD. Bogotá: Editorial del Ministerio de Salud de Colombia. 1991; 3-41.

7.- Álvarez Gómez M, Soria Aznar J, Galbe Sánchez-Ventura J: Importancia de la vigilancia del desarrollo psicomotor por el pediatra de atención primaria: revisión del tema y experiencia de seguimiento en una consulta en Navarra. Rev Pediatr Aten Primaria 2009; 11: 65-78.

8.- American Academy of Pediatrics Committee on Children with Disabilities: Developmental surveillance and screening of infants and young children. Pediatrics 2001; 108: 192-5.

9.- Figueiras A, Neves de Souza I, Ríos V, Benguigui Y: Manual para la vigilancia del desarrollo infantil en el contexto de AIEPI. Washington: Organización Panamericana de la Salud (OPS) 2006; 2-55.

10.- Salamanco G, D'anna $C$, Lejarraga $H$ : Tiempo requerido para la administracion de una prueba de pesquisa de trastornos del desarrollo psicomotor infantil. Arch Argent Pediatr 2004; 102: 165-9.

11.- Lira $M$, Gálvez $G$ : El programa de estimulación precoz diez años después de una experiencia piloto. En: Cusminsky M., Moreno E., Suárez-Ojeda N. eds. Crecimiento y Desarrollo. OPS. Publicación Científica Nro 510. 1988; 383-404.

12.- Bedregal $P$ : Instrumentos de medición del desarrollo en Chile. Rev Chil Pediatr 2008; 79: 32-6.

13.- Vargas NC, Quezada AL: Epidemiología, nueva morbilidad pediátrica y rol del pediatra. Rev Chil Pediatr 2007; 78: 103-10.

14.- Pérez Olarte P: Evaluación y manejo del niño con retraso psicomotor. Pediatr Integral 2003; VII: 557-66.

15.- Council on Children with Disabilities, Section on Developmental Behavioral Pediatrics, Bright Futures Steering Committee and Medical Home Initiatives for Children With Special Needs Project Advisory Committee: Identifying infants and young children with developmental disorders in the medical home: an algorithm for developmental surveillance and screening. Pediatrics 2006; 118: 405-20. 
16.- Schonhaut L, Álvarez J, Salinas $P$ : El pediatra y la evaluación del desarrollo psicomotor. Rev Chil Pediatr 2008; 79: 26-31.

17.- Bricker D, Squires J, Mounts L, Potter L, Nickel R, Farrell J: Ages and Stages Questionnaires (ASQ): A parent-completed child-monitoring system. Baltimore: Paul Brookes Publishing Company. 1995.

18.- Doig K, Macias M, Taylor C, Jeffery R, Craver J: The Child Development Inventory: A developmental outcome measure for follow-up of the high-risk infant. J Pediatr 1999; 135: 358-62.

19.- Glascoe FP: Parent's Evaluation of Developmental Status (PEDS). Nashville: Ellsworth \& Vandermeer. 1998.

20.- Squires J, Bricker D: Ages \& And Stages Questionnaires. Third Edition. (ASQ-3): A Parent-Completed ChildMonitoring System. Standford: Paul Brookes Publishing Company. 2009.

21.- Schonhaut L, Salinas P, Armijo I, Schönstedt M, Álvarez $J$, Manríquez M. Validación de un cuestionario autoadministrado para la evaluación del desarrollo psicomotor. Rev Chil Pediatr 2009; 80: 513-9.

22.- Fejerman N, Fernández Álvarez E: Neurología Pediátrica. Buenos Aires: Panamericana 2007; 25-31.

23.- Frankenburg $W$, Dodds $J$, Asrcher P, et al: Denver II. Training Manual. Denver: Denver Developmental Material. 1992; 34-42.

24.- Glascoe F, Byrne KE, Ashford L, Johnson K, Chang B, Strickland B: Accuracy of the Denver-II in developmental screening. Pediatrics 1992; 89: 1221-25.

25.- Wachtel R, Shapiro B, Palmer F, Allen M, Capute A: CAT/CLAMS: a tool for the pediatric evaluation of infants and young children with developmental delay. Clinical Adaptive Test/Clinical Linguistic and Auditory Milestone Scale. Clin Pediatr (Phila) 1994; 33: 410-15.

26.- Accardo P, Capute A: The Capute Scales. Cognitive adaptive test/Clinical linguistic and auditory milestone scale (CAT/CLAMS). Baltimore: Paul Brookes Publishing Company 2005; 44-63.

27.- Bayley N: Bayley Scales of Infant Development Manual. Nueva York: The Psychological Corporation. 1993; 67-77.

28.- Reiner Hess C, Papas M, Black M: Use of the Bayley Infant Neurodevelopmental Screener with an environmental risk group. J Pediatr Psicol 2004; 29: 321-30.

29.- Rodríguez S, Arancibia V, Undurraga C: Escala de Evaluación del Desarrollo Psicomotor de 0 a 24 meses.
Santiago de Chile: Galdoc 2001; 18-54

30.- Haeussler I, Marchant T: TEPSI. Test de Desarrollo Psicomotor: 2 a 5 años. Santiago de Chile: Universidad Católica de Chile 2007; 22-45.

31.- Lejarraga H, Kelmansky D, Pascucci M, Salamanco G: Prueba Nacional de Pesquisa, PRUNAPE, Manual técnico. Buenos Aires: Fundación Hospital Garran 2005; 19-136.

32.- Piaget J: El Nacimiento de la Inteligencia en el Niño. Buenos Aires: Crítica 1973; 13-35.

33.- Oiberman A, Mansilla $M$, Orellana $L$ : Nacer y Pensar. Escala Argentina de Inteligencia (EAIS) de 6 meses a 2 años. Buenos Aires: CIIPME-CONICET 2002; 2: 11-44.

34.- Atkin LC, Supervielle T, Sawyer R, Cantón P: Paso a paso: cómo evaluar el desarrollo y crecimiento de los niños. México: UNICEF/PAX. 1987; 12-23.

35.- Marinho H: Escala de Desenvolvimento. Rio de Janeiro: Soc Pestalozzi do Brasil. CENESP-MEC. 1977.

36.- American Academy of Pediatrics: Follow-up care of high-risk infants. Pediatrics 2004; 114: 1377-97.

37.- Figueiras ACM, Fiorini Puccini R, Koga da Silva EM, Marcondes Pedromônico MR: Avaliação das práticas e conhecimentos de profissionais da atenção primária a saúde sobre vigilância do desenvolvimento infantil. Cad Saúde Pública 2003; 19: 1691-9.

38.- Atkin LC: Analysis of instruments used in Latin America to measure psychosocial development in children from 0 to 6 years of age. Instituto Nacional de Perinatología México 1989; p. 1-25.

39.- Schapira IT: Comentarios y aportes sobre desarrollo e inteligencia sensorio-motriz en lactantes. Rev Hosp Mat Inf Ramón Sardá 2007; 26: 21-7.

40.- Lejarraga H: La pesquisa de problemas del desarrollo. La Prueba Nacional de Pesquisa. En: Lejarraga H. ed. Desarrollo del Niño en Contexto, Buenos Aires: Paidós. 2004; 341-78.

41.- Rydz D, Srour M, Oskoui M, et al: Screening for Developmental Delay in the Setting of a Community Pediatric Clinic: A Prospective Assessment of Parent-Report Questionnaires. Pediatrics 2006; 118: 1178-86.

42.- Tellerias L, Molina H: Desarrollo infantil temprano. Un desafío. Rev Chil Pediatr 2008; 79: 9-10.

43.- Aliaga Tovar J: Psicometría: test psicométricos, confiabilidad y validez. En: Quintana A., Montgomery W. eds. Psicología: Tópicos de Actualidad. Lima: UNMSM. 2006; 85-108. 